\title{
DRAMATIZACIÓN DEL CONOCIMIENTO: SIMULACIONES EN EL CONTEXTO DE LOS ESTUDIOS JURÍDICO-LABORALES DE GRADO Y DE POSGRADO ${ }^{1}$
}

\author{
JOSE M. MORALES ORTEGA \\ Profesor Titular del Derecho del Trabajo y de la Seguridad Social \\ jmmoraleso@uma.es \\ Universidad de Málaga
}

\begin{abstract}
Resumen
Las nuevas exigencias propias del Espacio Europeo de Educación Superior están obligando a unas imprescindibles adaptaciones en las formas de enseñar y de aprender y, sin duda, una de las técnicas más acertadas en esta dirección es la dramatización del conocimiento o, más conocida, como simulaciones, en las que los alumnos interpretan y adoptan posiciones y papeles, con los que colocarse en situaciones lo más cercanas posibles a la realidad. De este modo, se favorece un conjunto de competencias necesarias y adecuadas para su desarrollo profesional. Ahora bien, esta forma de aprendizaje se enfrenta, hoy por hoy, a numerosos problemas aplicativos derivados de los más diversos ámbitos pero, fundamentalmente, reducidos a los siguientes: el propio sistema educativo y las actitudes y disposiciones de profesor y alumno.
\end{abstract}

Palabras clave: Dramatización, simulación, competencias, aprendizaje, desarrollo profesional, práctica, pensamiento crítico y evaluación.

\begin{abstract}
The new demands of the European Space of Higher Education are forcing essential adaptations in the ways of teaching and learning and, without a doubt, one of the most successful techniques in this direction is the dramatization of knowledge or, better known, as simulations, in which students interpret and adopt positions and roles, with which to position themselves in situations as close as possible to reality. In this way, a set of necessary and appropriate skills for their professional development is favored. Now, this form of learning is facing, today, many application problems arising from the most diverse areas but, fundamentally, reduced to the following: the educational system itself and the attitudes of teacher and student.
\end{abstract}

Keywords: Dramatization, role playing, competitions, learning, professional development, practice, critica thinking and evaluation.

\footnotetext{
${ }^{1}$ Este trabajo se inserta en el marco de dos Proyectos de Innovación Educativa de la Universidad de Málaga, que son: PIE 15-105 "Aprendizaje colaborativo, método del caso y role-playing como metodología para la adquisición de las competencias profesionales necesarias en el ámbito jurídico en asignaturas de Grado y Master: el trabajo en equipo" y PIE 17-112 "La simulación como herramienta de enseñanza-aprendizaje práctico en el ámbito jurídico"

REJIE Nueva época: Revista Jurídica de Investigación e Innovación Educativa

Núm.17, Enero 2018, pp. 81-98

[En línea] http://www.revistas.uma.es/index.php/rejie
} 


\section{Sumario:}

1. Presentación del trabajo. 2. La necesidad del desarrollo de competencias de cara a la inserción laboral del joven universitario 3. Capacidades y competencias: otra forma de aprender y de enseñar 4. Simulaciones o dramatizaciones en asignaturas jurídicolaborales 4.1. Dinámica de las simulaciones o dramatizaciones. 4.2. La calificación de las simulaciones como parte de la evaluación del alumnado. 4.3. Objetivos, deficiencias y valoraciones de la dramatización del conocimiento. 5. Ejemplificación de las simulaciones en distintas asignaturas jurídico-laborales. 5.1. Simulación genérica: proceso de selección de personal. 5.2. Simulaciones específicas. 6. A modo de conclusiones

\section{Presentación del trabajo}

El Espacio Europeo de Educación Superior, fruto de la conocida Declaración de Bolonia, tiene, como es sabido, entre otros objetivos (Mayorga Fernández, 2010: 92): favorecer una educación y una formación, que faciliten el camino de acceso al mercado de trabajo. Y con ese propósito, los estudios universitarios, tanto de grado como de posgrado, tienen que diseñarse tomando en consideración las competencias y las habilidades, que faciliten la consecución de este objetivo. Competencias y habilidades que, obviamente, son muchas y muy variadas; y todas ellas, de una u otra forma, pueden tener encaje en el devenir del curso universitario. La cuestión se centra en encontrar las fórmulas más acertadas e idóneas de cara a que el alumno las adquiera. Una de ellas, sin duda, es la dramatización o simulación de situaciones, con las que acercar, en la medida de lo posible, al alumno a la realidad, por lo que aquí importa, socio-laboral. La adquisición, por tanto, de estas habilidades y competencias permitirá completar la formación del alumno al haberse enfrentado a situaciones de diferente orden $\mathrm{y}$ naturaleza, con las que desarrollar o potenciar capacidades, que en el aprendizaje tradicional han sido, cuando menos, infravaloradas.

Todo lo anterior es coherente con las exigencias del mercado de trabajo. Éste ya no busca, exclusivamente, alumnos universitarios con una formación circunscrita a la adquisición de unos conocimientos teóricos que, sin deberse desprestigiar, deben ser completados con la potenciación de ciertas facultades cada vez más reclamadas por las empresas. En consecuencia, la universidad no puede, ni debe permanecer al margen de esa realidad del mercado de trabajo si se quiere generar auténticos profesionales.

Con este objetivo, y siempre siendo conscientes de las limitaciones de distinto orden que existen en el desarrollo de los cursos universitarios -y sobre las que se volverá en otro momento-, se pueden incorporar a los mismos actividades orientadas a lograr, como mínimo, que el alumno adquiera, en un primer momento, un conocimiento lo más exacto posible de la realidad a la que se va a enfrentar; en segundo lugar, ayudarle a potenciar o encauzar esas habilidades, en muchas ocasiones innatas, necesarias en su desarrollo profesional; y por último, dotarlo y enseñarle las herramientas y los instrumentos oportunos para su consecución. No se puede ocultar que se trata de una tarea sumamente compleja para el profesor pero, al mismo tiempo, muy enriquecedora cuando se logra el resultado final. 
A lo largo de estas páginas, se va a intentar, fundamentalmente, transmitir la experiencia de muchos años de docencia -por tanto, no se inicia con Bolonia- llevando a cabo un conjunto de actividades orientadas a intentar lograr una formación más completa del alumno. Actividades que, como es lógico, se han canalizado de manera distinta pero, muy significativamente, a través de las simulaciones o dramatizaciones. Así pues, se va a estructurar el trabajo del siguiente modo. Se va a comenzar con una introducción más teórica, que responde a la necesidad de incorporar, como una actividad importante y necesaria en los estudios de grado y de posgrado, estas simulaciones para, a continuación, proceder a describir y comentar la dinámica y tipos de estas simulaciones para terminar con las especificidades propias de las asignaturas, que he impartido en los últimos años. Todo ello sin olvidar los problemas suscitados en cada apartado, los cuales no deben disuadir al docente de su realización, ya que son muchas más, en la generalidad de las ocasiones, sus ventajas para el desarrollo del curso, para el alumno y, por supuesto, para el profesor.

\section{La necesidad del desarrollo de competencias de cara a la inserción laboral del joven universitario}

Como se ha anticipado, con las simulaciones o dramatizaciones se pretende que el alumno adquiera un conjunto de competencias y habilidades, con las que allanar su acceso al mercado de trabajo -y, por qué no, favorecer su desarrollo, incluso, personal-.

Tradicionalmente, la formación ha estado vinculada a la adquisición de unos conocimientos teóricos en cualquiera de los diferentes niveles formativos. Sin embargo, esta idea se presenta, en la actualidad, anticuada o, si se prefiere, no acorde con las demandas de los empleadores. Hoy en día, no es suficiente una titulación sino que además hay que poseer unas competencias y capacitaciones que exceden, en muchos casos, la formación entendida en sentido clásico. De ahí que no resulte extraño, aunque sea un campo ajeno al ámbito estrictamente educativo, que la Ley Orgánica 5/2002, de 19 de junio, de las Cualificaciones y de la Formación Profesional (BOE de 20 de junio), en su art. 7.3, distinga entre cualificación y competencia profesional. La primera, se entiende como "el conjunto de competencias profesionales con significación para el empleo que pueden ser adquiridas mediante formación modular $u$ otros tipos de formación a través de la experiencia laboral'. Mientras que la competencia profesional es "el conjunto de conocimientos y capacidades que permitan el ejercicio de la actividad profesional conforme a las exigencias de la producción y el empleo". Y es a esa cualificación y competencia hacia donde deben dirigirse también los esfuerzos docentes con la finalidad de no restar oportunidades de inserción laboral al estudiante universitario.

En este mismo orden de ideas, hay que llamar la atención sobre el hecho de que las Orientaciones Integradas Europa 2020, aunque en materia de empleo, convierten también la formación, en su más amplia acepción, en la base imprescindible de acceso al mercado de trabajo. Y, de manera muy resumida, se quiere indicar que el espíritu de dichas Orientaciones -por tanto, de recomendaciones a los Estados miembros, en este caso, con la participación de los interlocutores sociales- se asienta sobre los siguientes pilares: a) formación acorde con las exigencias presentes y futuras del mercado de trabajo, b) aprendizaje permanente, c) reconocimiento de competencias, d) adquisición de competencias transversales, e) favorecer la formación de los que carecen de ella o la tienen anticuada -en particular, los jóvenes-, al tiempo que se potencia la de los que 
tienen más altas cualificaciones, f) mejora de los sistemas educativos, g) movilidad en los procesos de aprendizaje, h) lucha contra el fracaso escolar, i) itinerarios flexibles de aprendizaje y j) formación en alternancia. En definitiva, todo un conjunto de directrices, que deben marcar el correcto aprendizaje del alumno en general, y del universitario en particular.

Pues bien, esa significación de las competencias y de las habilidades se hace especialmente reseñable cuando se observa que en los procesos de selección de personal, estas competencias están adquiriendo mucho más protagonismo, debido al enjuiciamiento por los oportunos sicólogos, que los propios conocimientos teóricos y prácticos aprendidos en la formación reglada. En esta dirección, por ejemplo, juega un papel importante las simulaciones de los procesos de selección, que deberían ser comunes en todos los grados y posgrados. Es más, deberían formar parte del aprendizaje obligatorio de todo alumno universitario. El hecho de que el alumno se enfrente, aunque sea a través de las simulaciones, a esa realidad se convierte en un instrumento de gran valor para su aprendizaje.

En principio, la planificación universitaria no ha sido ajena a dichas competencias y habilidades; tanto es así que, en el marco del Espacio Europeo de Educación Superior, se hace obligatorio, a la hora de elaborar las programaciones docentes, incluir, como es sabido por todos, las competencias que va a desarrollar el alumno en la correspondiente asignatura. Ahora bien, éstas presentan una gran deficiencia consistente en que su diseño y determinación queda en manos de las instancias universitarias competentes; sin que se cuente con la presencia de los interlocutores sociales y, muy particularmente, de la clase empresarial. Su participación permitiría una concreción de las competencias mucho más realista y acorde con las necesidades de las organizaciones. Al no hacerse, muchas de dichas competencias, capacidades y habilidades quedan como meras formulaciones esteriotipadas, que hacen dudar de que el sistema universitario, en realidad, capacite al alumno, al menos en este aspecto, para su inserción laboral.

Aún con esta desesperanzadora afirmación, el hecho de que estas competencias ya sean tenidas en cuenta, y vayan interiorizándose, con todas sus deficiencias, en la práctica docente suponen un enorme adelanto para la enseñanza universitaria y, sobre todo, para uno de sus objetivos: la integración laboral del alumno. Pues nunca puede olvidarse que, de acuerdo con el art. 1.b) de la Ley Orgánica de Universidades 6/2001, de 21 de diciembre (BOE de 24 de diciembre), entre las funciones de la universidad, está "la preparación para el ejercicio de actividades profesionales que exijan la aplicación de conocimientos y métodos científicos y para la creación artística".

En suma, el mercado de trabajo reclama, y cada vez lo hará con más énfasis, un personal cualificado y competente conforme a las coordenadas de la clase empresarial. Y para ello se hace imprescindible que el alumno universitario adquiera una formación profesionalizada entendiendo por ésta tanto la teórico-práctica derivada de su grado o postgrado como la vinculada a las tan reiteradas competencias, habilidades $\mathrm{y}$ capacidades; y ambas complementadas con una adecuada orientación laboral.

La orientación laboral es, sin duda, una de las grandes quiebras de nuestro sistema educativo. La ausencia de una correcta orientación laboral tiene, como es previsible, significativas consecuencias a la hora de la inserción laboral del joven. Su desconocimiento de la realidad del mercado de trabajo -índices de inserción laboral, 
tasas de desempleo, salidas profesionales, requerimientos formativos, oposiciones, y así un largo etcétera-, le puede conducir, fundamentalmente, a optar por salidas formativas -sea en el paso del bachiller al grado o de éste al postgrado- carentes de todo futuro laboral. Ello se hace particularmente problemático en el caso de las mujeres. Éstas suelen decantarse por itinerarios formativos, que acusan unos mayores índices de desempleo y que, curiosamente, están vinculados con su rol en la sociedad. Cuesta trabajo entender que esta opción sea fruto de una tendencia consustancial a la mujer, por lo que hay que aceptar que es fruto de una educación sexista no contrarrestada por una acertada orientación laboral. Y ello pese a que el art. 42 de la Ley Orgánica 3/2007, de 22 de marzo, para la igualdad efectiva de mujeres y hombres (BOE de 23 de marzo) afirme que "se mejorará la empleabilidad... potenciando su nivel formativo y su adaptabilidad a los requerimientos del mercado de trabajo".

Todo lo anterior reclama nuevas metodologías docentes construidas, principal aunque no exclusivamente, sobre un mayor activismo e interacción entre las dos partes implicadas. Esta renovación metodológica es, sin duda, el gran reto de la pedagogía del siglo XXI (Ortíz de Urbina Criado, Medina Salgado y De la Calle Durán, 2010: 279).

\section{Capacidades y competencias: otra forma de aprender y de enseñar}

Como se acaba de indicar, la incorporación en el aprendizaje de estas competencias tales como el trabajo en equipo, la capacidad de reacción ante situaciones imprevistas, las exposiciones argumentadas, etcétera- exige de un esfuerzo por parte de todos los sujetos implicados, ya que supone un cambio de mentalidad. Y ello se tiene que producir mediante, por un lado, la concienciación de la efectividad de las mismas para el desarrollo formativo y profesional del alumno, y, por otro, la dotación de los medios necesarios para poder potenciar y desarrollar dichas capacidades y habilidades.

Comenzando con el cambio de mentalidad y reduciendo éste a los dos protagonistas profesor y alumno-, dicha renovación reclama cambios por ambas partes; sin embargo, curiosamente, tanto alumnos como profesores suelen estar más cómodos en los métodos antiguos y posiciones pedagógicas pasadas que, no por ello, deben ser rechazados de plano. Todo lo contrario. Debe aprovecharse lo positivo o, si se prefiere, integrarse en esos métodos y posiciones la renovación, ya que los mismos también han dado resultado -y no deben ni ocultarse, ni obviarse- a lo largo del tiempo. Dicho ello, hay que continuar indicando que ese cambio debe estar construido, principalmente, sobre la base de un comportamiento proactivo de alumnos y profesores (Taléns Visconti, 2016: 205 y Mayorga Fernández y Madrid Vivar, 2010: 98).

En el caso de los alumnos, se requiere de su mayor implicación; de tal modo que abandonen la dinámica habitual, por todos conocida, de limitarse a copiar apuntes y examinarse al final del curso (Ortíz de Urbina Criado, Medina Salgado y De la Calle Durán, 2010: 282) por otra, en la que acepte que, además de receptor, es emisor y artífice de su aprendizaje mediante el desarrollo de sus capacidades -además, lógicamente, del conocimiento de la correspondiente rama del saber-. Ello obliga a un trabajo continuo y constante a lo largo del curso, que se va a traducir en investigación, búsqueda y, fundamentalmente, razonamiento y crítica. De asumir todo ello, el alumno se sentirá -o se le hará sentir- protagonista de su aprendizaje y de su formación. 
En el caso de los profesores, se necesita del manejo de un conjunto de herramientas, con las que conseguir, en primer lugar, la descrita implicación; o sea, que el objetivo fundamental del profesor tiene que ser la motivación del alumno. Conseguido éste, lo que no siempre es fácil, se iniciará el camino a la potenciación de sus capacidades, competencias y habilidades. Ello se puede alcanzar a través de distintas medidas; aunque lo ideal es que confluyan todas ellas en cada uno de los cursos. Antes de continuar, se quiere precisar que no siempre la responsabilidad es exclusiva del docente, ya que, en más ocasiones de las deseadas, los alumnos no son receptivos, ni permeables a las propuestas y orientaciones del profesor por mucho que sea su esfuerzo.

Dichas medidas se pueden concretar en tres. En primer lugar, debe realizarse un cambio en el planteamiento de la formación teórica. En los últimos años, existe una tendencia dirigida a desprestigiar la histórica clase magistral cuando, realmente, ésta sólo requiere de una transformación en su planteamiento. En efecto, el entendimiento de la clase teórica -como aquella para hacer conocer a los alumnos los contenidos de la correspondiente asignatura- como el monólogo del profesor resulta disfuncional a efectos pedagógicos. Ahora bien, ese tipo de clase que, desde aquí, se considera imprescindible y fundamental, puede ser reinventada mediante un instrumento tan básico como el diálogo con el alumno -lo que también favorece determinadas competencias de éste-; es más, ello es la premisa de partida del principal, y casi único, cometido del profesor: fomentar el razonamiento crítico del alumno. Para ello, basta con crear un ambiente distendido favorecedor de la participación del discente; al tiempo que hay que aportarle los conocimientos necesarios así como las herramientas suficientes para alcanzar dicho objetivo -no obstante, debe aclararse que no se está defendiendo en este momento esa otra corriente pedagógica basada casi exclusivamente en convertir al alumno en el responsable de su aprendizaje, al limitarse el profesor a darle las oportunas herramientas pues, de seguirse esta tendencia, el resultado no será otro que el autoservicio del aprendizaje-. Por tanto, el esquema de este tipo de clases sería la presentación y explicación del tema y la aportación de herramientas e información y, todo ello, desde el diálogo y la crítica razonada. Esto esfuerzo del profesor, sin ningún género de dudas, se debe traducir en el reconocimiento y prestigio de su labor docente.

En segundo lugar e íntimamente conectado con lo anterior, debe actuarse sobre el alumnado a fin de conseguir, fundamentalmente, su motivación e interacción durante las clases, sean teóricas -en la concepción expuesta anteriormente- o prácticas -dentro de las cuales hay que incluir las simulaciones-. Hay que hacerlas atractivas y, para ello, una herramienta clave es hacerle comprender la importancia, actualidad y repercusión en su desarrollo profesional y personal de la asignatura. No se puede negar que ello depende, en gran medida, de la propia asignatura pero, incluso, en las que pueden parecer más alejadas de tales objetivos, siempre es posible encontrar puntos de anclajes para lograr esa implicación del alumno.

En mi caso, dicha implicación es relativamente fácil, puesto que, salvo muy concretas ocasiones, la mayoría -por no decir la totalidad- de los alumnos tienen que insertarse laboralmente, por lo que el conocimiento de sus derechos y de sus obligaciones laborales así como del mundo de las relaciones de trabajo facilita que los alumnos vean la utilidad, la actualidad y la importancia de cara a su desarrollo profesional de las correspondientes asignaturas. 
Ahora bien, siempre hay que atender a las características de la clase, puesto que en la consecución de tales objetivos va a influir, poderosamente, sus peculiaridades sin que se pueda, por tanto, diseñar, ni programar de antemano las actividades del curso, puesto que, de hacerse, ello conducirá, con mucha frecuencia, al fracaso. Así pues, lo más acertado es conocer a la clase -se acepta que en el número limitado y escaso de clases en los grados y, mucho más, en los posgrados dificulta este conocimiento; no obstante, siempre es factible posponer dicha programación, con carácter orientativo, a las primeras clases del curso y, con posterioridad, diseñar, planificar y realizar las oportunas actividades tanto teóricas como prácticas. Y siempre de una manera ni rígida, ni inflexible; de tal manera, que pueda ir adaptándose a las necesidades de la clase. Por esta razón, se considera totalmente antipedagógico la realización de los cronogramas sin tenerse conocimiento de las características del curso.

En tercer lugar y con todo lo anterior, debe acudirse a un abanico de actividades potenciadoras de las capacidades, habilidades y competencias tanto del conjunto del alumnado como de cada alumno en particular. En esta dirección, y junto a la indicada formulación de las "clases teóricas" -se quiere reiterar que no hay que darle un contenido peyorativo a las mismas, siempre y cuando se consiga esa descrita interacción del alumno-, las actividades tienen que responder a dicha exigencia. Ante la dificultad de encontrar una actividad comprensiva de todas esas habilidades y competencias es por lo que tienen que diseñarse varias a lo largo del curso. No obstante, hay que admitir que las simulaciones o dramatizaciones son, con toda seguridad, una de las actividades más completas.

Pero antes de entrar en el análisis de dichas simulaciones, se quiere, aunque sea a vuela pluma, plantear, como mínimo, algunos -otros ya han sido esbozados- de los principales problemas, a los que se enfrenta el docente a la hora de poner en práctica toda esta metodología docente.

Fundamentalmente, los problemas son de una doble naturaleza: tiempo y medios. Por lo que respecta al tiempo, la reforma del sistema universitario ha empobrecido, muy llamativamente, la posibilidad de realizar un aprendizaje completo y compresivo de todas las necesidades de los alumnos. Resulta, normalmente, ridículo el número de horas de clases, lo que ha obligado tanto a reducir temarios como a eliminar actividades. Y ello es totalmente contradictorio con esa misma pretensión del sistema de atender a un mayor número de requerimientos tanto para el docente como para el discente. Mientras que en relación con los medios, éstos no siempre existen o no siempre se acomodan a las necesidades del curso. Sin entrar, por supuesto, en la inobservancia de la ratio alumno-profesor.

\section{Simulaciones o dramatizaciones en asignaturas jurídico-laborales}

En este apartado se va a presentar la dinámica, objetivos y deficiencias encontradas en las simulaciones realizadas a lo largo de los años, lo que ha permitido ir corrigiendo los errores al tiempo que ir incidiendo en aquellos aspectos que mejor se adecuan a los objetivos de las mismas. No obstante, se va a centrar la atención, por razones de actualidad, en los tres últimos años y, más exactamente, en tres asignaturas de grado Solución Extrajudicial de Conflictos de Trabajo del Grado de Relaciones Laborales y Recursos Humanos, Relaciones Laborales en la Empresa del Grado de Derecho y Legislación Laboral en el Sector Turístico del Grado de Turismo- y una de posgrado - 
Mediación Laboral perteneciente al Master de Mediación-. Junto con estas simulaciones específicas por razón de la asignatura se ha practicado en los diferentes grados, en los que se imparte docencia una simulación, cuyo contenido es un proceso de selección de personal.

\subsection{Dinámica de las simulaciones o dramatizaciones}

Se va a distinguir entre la dinámica de las simulaciones de las asignaturas que, aunque distintas, en esencia, presentan un idéntico tratamiento, y las simulaciones, cuyo contenido es el proceso de selección.

a) Dinámica de las asignaturas jurídico-laborales

Como es natural, estas simulaciones se desarrollan como una actividad de carácter grupal; y así se favorece el trabajo en equipo o, si se prefiere, el trabajo colaborativo, lo que es cada vez más valorado por las empresas.

Al realizarse más de una simulación durante el curso -como mínimo, dos-, los grupos se constituyen de dos formas distintas. En una ocasión, por voluntad de los alumnos; de tal modo, que son ellos los que deciden quiénes los conforman. Mientras que en otra es el profesor el encargado de hacerlo. En este último caso, se intenta un doble objetivo. Por un lado, que con la adscripción del alumno al grupo se atienda a sus características y necesidades. Esto es, que se neutralice o relativice, en unos supuestos, y, en otros, que se potencie o fomente sus habilidades y características. Entre ellas, por ejemplo, la timidez, la espontaneidad, la capacidad de respuesta o la propia capacidad de trabajo en equipo. Y por otro lado, que no exista ninguna conexión, ni relación entre los alumnos. Esta decisión es resultado de que se busca un acercamiento al mundo real en tanto en cuanto, en sus futuras profesiones, tendrán, en muchísimos puestos de trabajo, que trabajar y formar equipos con otras personas que, necesariamente, no tienen por qué ser de su círculo amistoso -y, por consiguiente, relaciones contaminadas- o, si se quiere, de buenos compañeros. Así, se favorece la aproximación del alumno a la realidad del mercado de trabajo.

La experiencia ha puesto de manifiesto que los alumnos prefieren la primera opción pero, para el profesor, es mucho más efectiva la segunda, ya que le permite, en primer lugar, contrarrestar las deficiencias derivadas de cuando la elección compete, en exclusiva, a los alumnos, consistentes en reiterar una misma forma de trabajo y un mismo reparto de "competencias", y, en segundo lugar, la verdadera capacidad para trabajar en equipo. Con la corrección de ambas cuestiones, se pone a prueba la adaptación del alumno a diferentes circunstancias y a distintos compañeros de trabajo.

Con independencia de la forma de conformación del grupo, se intenta que el número máximo de miembros del equipo sea de 3. Aunque, como es lógico, ello va a depender del número de alumnos en clase que, con demasiada frecuencia, es lo suficientemente alto como para obligar a ampliar el número pero nunca por encima de 5.

Otra de las competencias que se fomentan con este tipo de actividad es la asunción de diferentes roles, lo que obliga al alumno a situarse en posiciones no necesariamente acordes con sus pensamientos o ideologías. Ello, en el ámbito jurídico-laboral, es especialmente significativo. Por este motivo y para evitar posiciones acomodaticias de 
los alumnos, el profesor es el que reparte los papeles para intentar, en la medida de lo posible, adjudicar los roles más alejados a las características de los alumnos (Ortíz de Urbina Criado, Medina Salgado y De la Calle Durán, 2010: 284). Como siempre, lo que se intenta es situar al joven lo más cercanamente posible a la realidad.

Por el contenido de las asignaturas, la asunción de roles suele estar fácilmente predeterminada; lo normal, es que se trate de trabajador -sea individualmente, sean los representantes de los trabajadores o sean los sindicatos- y de empleador -o sus organizaciones empresariales-. Lo que supone el debate dialéctico entre ambas partes de las relaciones laborales. Estas posiciones se complementan con la intervención de otros sujetos; unos, normalmente obligatorios, como es la presencia de un tercero dirimente en unos supuestos, el órgano jurisdiccional, y, en otros, un órgano extrajudicial de solución de conflictos (conciliador/es, mediador/es o árbitro/s)-; y otros, dependiendo del supuesto de hecho, la administración laboral o las asociaciones de ciudadanos o comerciantes.

No todos los supuestos de hecho implican al mismo número de grupos. Así, hay casos, en los que son dos grupos -trabajadores y empleadores-; otros, tres grupos -trabajadores, empleadores y órgano dirimente-, y, en una tercera ocasión, a éstos se añaden, como se ha señalado, otros colectivos o autoridades públicas.

Designados los grupos y los roles, se les da a los alumnos el supuesto de hecho sobre el que va a versar la oportuna simulación; y que se caracteriza por las siguientes notas: abierto y breve -con la finalidad de que los alumnos puedan agudizar el ingenio y, al mismo tiempo, generar la posibilidad de que cada uno de los grupos, sobre una premisa básica, puedan, como en la realidad, utilizar las correspondientes argumentaciones desconocidas por el grupo contrario-, real o ficticio -aunque existe una preferencia por los ficticios debido a que, en caso contrario, el alumno, en lugar de buscar las argumentaciones jurídicas oportunas, emplea su tiempo en buscar la solución dada, normalmente, por los órganos jurisdiccionales o extrajudiciales-; y sin indicaciones, ni orientaciones -con la intención de que sean capaces de extraer del relato fáctico aquellos elementos con relevancia jurídica-.

El supuesto de hecho lo conocen los alumnos con la suficiente antelación para su preparación. La antelación va a depender de dos factores. En primer lugar, de la duración del curso. No es lo mismo un semestre de un grado que un módulo de un master pues, aunque en ambos, el supuesto es conocido por el alumno desde el primer día de clase, la fecha de la realización difiere considerablemente. En el caso del grado, hay un mayor espacio temporal, lo que facilita la labor del alumno al tener un mayor tiempo para la preparación; mientras que en el caso del master, ese espacio es bastante más reducido. De hecho, suele ser una de las principales críticas realizadas por los alumnos, puesto que no tienen demasiado tiempo ni para prepararlo, ni para asimilar los conocimientos pertinentes.

En contadas ocasiones, el profesor acompaña al supuesto de hecho un pequeño dosier sobre la cuestión conflictiva. Ello sucede, principalmente, con los alumnos, que no están cursando un grado o un posgrado de contenido estrictamente jurídico -por ejemplo, en los masteres de mediación o de igualdad-. Las deficiencias jurídico-laborales de estos alumnos obliga a esta ayuda complementaria, lo que no anula, por supuesto, que tengan que realizar una búsqueda de información y documentación adicional. Búsqueda que, en 
las restantes ocasiones, compete en exclusiva al alumno en particular, y al grupo en general.

Con todo ello, el alumno y el grupo procederán a preparar la correspondiente simulación o dramatización. Durante esta fase, el profesor no aportará ayuda suplementaria a ninguno de los grupos de cara a que sean ellos mismos los verdaderos y únicos artífices de la preparación.

Llegado el momento de la realización, los grupos intervienen durante un período de tiempo, que depende del disponible en cada asignatura. Normalmente, se suele establecer una media hora por dramatización -no por grupo-. Durante este tiempo, los alumnos simularan la oportuna actividad; y, lógicamente, la dinámica difiere en función de dicha actividad.

En los casos de negociación de un convenio colectivo o de un acuerdo de empresa existe una mayor libertad formal. Suele iniciarse con una plataforma reivindicativa elaborada por el grupo de los trabajadores. Este documento se presenta a la contraparte con una antelación a la simulación de 3 días. De tal forma que el día de la realización de la simulación, dicha plataforma se convierte en el pistoletazo de salida a la misma. A partir de ese momento, el debate y el diálogo -sin indicaciones, ni guiones preestablecidos pero sí como es normal con la correspondiente preparación- serán los protagonistas de la actividad.

En los casos de vía judicial o extrajudicial, de forma parecida al supuesto anterior, la parte que inicia el procedimiento presentará con una antelación, igualmente de 3 días, a la otra parte el escrito de iniciación -sea en forma de demanda o de escrito conforme a los requerimientos contenidos en los acuerdos marco de solución extrajudicial de conflictos-. El grupo que asume el papel de tercero dirimente tiene que llevar a la simulación una propuesta de sentencia o de acta, que, por lógica, puede ser modificada durante el transcurso de la simulación de conformidad con los argumentos $\mathrm{y}$ circunstancias, que se vayan esgrimiendo durante la misma.

En estos dos supuestos, el alumno desconoce, salvo las de inicio, las argumentaciones de la contraparte, lo que permite fomentar sus capacidades de improvisación. Ello ofrece la oportunidad de desarrollar roles distintos y posicionarse en posturas contrarias o distantes del planteamiento inicial del alumno e, incluso, de que tengan que ir cambiando de posicionamiento y argumentación a lo largo del desarrollo de la simulación.

En los casos de los procesos de selección, no hay nada conocido por el alumno; más que la oferta de empleo. Los alumnos deben aportar su currículo con una antelación de una o dos semanas -según el curso y el año-. El día de la simulación se comenzará con la designación de los alumnos, que han superado la criba curricular; y a partir de ahí, como se describirá más tarde, se procederá a la presentación de los distintos candidatos y de sus currículos, dinámicas de grupo y entrevistas.

Ni que decir tiene que las simulaciones implican un concreto número de grupos, lo que conlleva a que los alumnos no participantes puedan adoptar una actitud pasiva, fruto de su desconexión, durante la actividad. O sea, pueden mostrar desinterés o, sencillamente, desatención y aburrimiento. Para evitar este desafortunado resultado, se ha incorporado 
a las simulaciones dos actividades complementarias finales: debate -al no ser posible la interacción durante la simulación para no distorsionar su desarrollo- y cuestionario dirigido a los alumnos no participantes -se les comunica la existencia del mismo pero no su contenido con la finalidad de que los alumnos-público estén atentos a todas las cuestiones, que puedan suscitarse durante la simulación-. Así, todos ellos se implican en la dramatización ya sea como protagonistas, ya sea como público activo.

Para que dichas actividades finales puedan realizarse de manera correcta, se recomienda a los alumnos-público que realicen las necesarias anotaciones, para que les sirvan de cara a formular las críticas u observaciones a los participantes en la simulación así como para responder al cuestionario.

No es necesario entrar, por razones obvias, en el contenido del debate, que será alentado y fomentado por el profesor; pero sí en el cuestionario. Éste se compondrá de tres tipos de preguntas. Unas primeras, referidas a cuestiones de fondo y de forma sobre la actividad -quiebras argumentales, errores jurídico-laborales, etcétera-, lo que obliga a los alumnos a tener que estar sumamente atentos y asimilar conceptos y posturas; unas segundas, sobre qué grupo y qué alumno han llevado a cabo mejor la simulación -ni que decir tiene que no puede optarse por el grupo del que se sea parte, ni, por supuesto, el alumno puede elegirse a sí mismo; y ello, porque todos los alumnos de clase son, en unos momentos, protagonistas de la simulación, y, en otros, alumnos-público-, los más valorados en sendas categorías obtendrán una nota adicional a la del profesor, lo que favorece la competitividad y el esfuerzo de los participantes y, a la par, la crítica y la capacidad de evaluar de los alumnos-público; y unas terceras, relativas a la propia actividad en el sentido de que formulen las consideraciones oportunas sobre las ventajas y desventajas de estas simulaciones, lo que se traduce en una valoración y unas propuestas de mejora de este sistema de aprendizaje tras la identificación, de existir, de los problemas, que pueda originar esta actividad.

\subsection{La calificación de las simulaciones como parte de la evaluación del alumnado}

Como se ha anticipado, las simulaciones forman parte de un conjunto de actividades de distintas naturalezas. Cada una de ellas tiene una nota; y la suma de todas conforman la nota complementaria del alumno -que se suma a la del examen- resultado de una evaluación continua. Así pues, la calificación de la simulación sólo es, simplemente y con todo, una nota más, que evalúa determinadas competencias, capacidades y habilidades de los alumnos. Y ello, puesto que esta dramatización del conocimiento, con ser un instrumento muy adecuado y completo para el aprendizaje y la formación, no cubre todos los aspectos y facetas de los mismos. De ahí, se insiste, que haya que completarlo con exámenes, otras actividades prácticas, asistencia y participación en clase, etcétera, que permitan al profesor contar con todos los elementos de juicio necesarios.

Dicho lo cual, hay que continuar indicando una serie de observaciones en torno a la calificación de estas simulaciones.

En primer lugar, que las notas son individuales, y no del grupo. De este modo, se consigue un doble propósito. Por un lado, que todos los alumnos participen de manera activa; por ejemplo, ello es especialmente relevante de cara a la competencia de la expresión oral. En la medida en que no hay que entregar, salvo indicación del profesor, 
el expediente por escrito, la calificación toma en consideración las diferentes participaciones. Siendo así, los alumnos no intervinientes no pueden ser calificados. Por consiguiente, se les obliga a intervenir. Y, por otro lado, que la nota del grupo no esté supedita a la incorrecta o deficiente actuación de alguno de sus miembros (Taléns Visconti, 2016: 207).

En segundo lugar, que no cabe la autoevaluación, lo que no significa, y como se ha dicho al tratar el cuestionario final, que los restantes compañeros no valoren las actuaciones tanto de los grupos como de los alumnos individualmente considerados. De hecho, tienen que expresar su opinión explicando los motivos que le han conducido a la oportuna calificación. Ésta es un porcentaje sobre la calificación final de esta actividad por parte del profesor.

En tercer lugar, que la calificación puntúa tanto la documentación presentada -cuando sea necesaria y requerida- y el acto en sí de la simulación $-25 \%$ y $75 \%$, respectivamente, de la nota-. En el caso de no ser necesaria ninguna documentación, el 100\% de la nota será la intervención en la simulación. No obstante, se recomienda al grupo la elaboración de un informe jurídico -correspondiente al rol que ha tenido que ocupar en la simulación- para aquellas ocasiones, en las que el profesor no tiene clara la calificación; y así pueda contar con un elemento de juicio adicional.

Este planteamiento difiere, por sus particulares características, en el supuesto del proceso de selección. En esta ocasión y con la finalidad de motivar al alumno, la calificación, dependiendo del peso que tenga sobre el total de su nota final del curso, se reparte en función de las pruebas superadas. Normalmente, como ya se ha descrito, las fases son: criba curricular, presentación de currículo, dos dinámicas de grupo y entrevista personal. En suma, cinco pruebas. La superación de cada una de ellas supone un punto para el alumno; alcanzando la máxima calificación el candidato, que "obtenga" el correspondiente puesto de trabajo.

\subsection{Objetivos, deficiencias y valoraciones de la dramatización del conocimiento}

Los objetivos alcanzados con las simulaciones se pueden cifrar en los siguientes:

a) Adaptar los conocimientos a las competencias exigidas por los grados y posgrados y, al mismo tiempo, por el mercado de trabajo; de tal modo que se facilite su desarrollo profesional.

b) Fomentar las siguientes habilidades -todas ellas constadas y contrastadas con la experiencia docente-: trabajo en equipo -también conocido como cooperativo (Taléns Visconti, 2016: 206 y 208) o colaborativo (Ortíz de Urbina Criado, Medina Salgado y De la Calle Durán, 2010: 283)-, búsqueda de fuentes e información, expresión oral, asunción de roles, capacidad de debate, de reacción, de improvisación y de argumentación, pensamiento crítico (García Barrera, 2010: 3) y sujeción a trámites y procedimientos. Una forma de aprendizaje, por consiguiente, que integra diferentes competencias de gran significación para el desarrollo profesional y académico del alumno. 
c) Ni que decir tiene que el desarrollo de esta actividad requiere, por parte del alumno, de una enorme implicación y responsabilidad pero, de conseguirse, el alumno se siente protagonista de su desarrollo formativo al tiempo que aprende "divirtiéndose".

Por su parte, las deficiencias, no insalvables, son tres. En primer lugar, que es necesaria un previa formación teórica, lo que queda dificultado en los postgrados por el escaso tiempo existente, en la mayoría de las ocasiones, para impartir dicha formación, que es la base del aprendizaje práctico desarrollado a través de las simulaciones. De superarse este óbice, se consigue el conocimiento teórico-práctico de la asignatura.

En segundo lugar, que los alumnos no siempre entienden esta actividad -muy distinta del aprendizaje tradicional- como un elemento clave para su desarrollo formativo, por lo que si no se implican -lo que ocurre en ciertas ocasiones- puede que la simulación no aporte los resultados pretendidos. Ahora bien, cuando existe responsabilidad y seriedad por parte de los alumnos, el resultado es muy valorado por la clase.

En tercer lugar, que los alumnos suelen poner de manifiesto que la principal falla de estas simulaciones es la falta de tiempo tanto para su preparación -esta deficiencia se da casi exclusivamente en los postgrados- como para su realización. Mucho más problemática es esta segunda limitación para los casos, bastante habituales, en los que hay que realizar varias simulaciones -por la existencia de muchos grupos en el cursocada una de las cuales requiere, por tanto, de su tiempo; y no siempre es posible, con la importantísima reducción del número de clases en la enseñanza universitaria, dedicar varias de ellas a estas simulaciones, puesto que va en detrimento del resto de actividades y, por supuesto, del temario. Fruto de esta limitación, se suele ofertar la simulación del proceso de selección fuera del horario de clase, ya que se trata de una actividad que, como mínimo, necesita de 4 horas continuadas para su realización.

Pese a estas deficiencias, la valoración por parte de los alumnos es altamente positiva. $\mathrm{Y}$, a estos efectos, se quiere transcribir algunas de las opiniones vertidas por los mismos en el cuestionario final. No se trata de una elección intencionada pues, como ya se ha comentado, lo normal es que, como mínimo, el 95\% del alumnado elogia esta actividad.

Así, en el cuestionario se destaca que es una forma de aprendizaje mucho más fructífera que la estricta formación teórica -"estoy convencido de que estas simulaciones sirven más que las clases teóricas, no ya porque éstas no sirvan como método de enseñanza, sino porque en la simulación se plantean cuestiones reales por personas, que representan intereses contrapuestos" o "la simulación final me ha parecido la mejor práctica que he hecho, ya que se aprende de una forma diferente y mucho más entretenida, lo que hace que los conceptos calen mejor en los alumnos. Se aprende más en un día de este tipo de práctica que en cinco de teoría"-, que supone un acercamiento a la realidad -"en un futuro puede que nos encontremos ante este tipo de situaciones y, en ningún momento, nos han enseñado como son estos procesos. De ahí que esta práctica me haya gustado porque además de aprender como es una mediación nos ha permitido poner en práctica todos los conocimientos adquiridos" o "realizar este tipo de simulaciones/prácticas es lo que permite obtener experiencia y conocimientos, ya que nos pone en situaciones que nunca hemos vivido"-, que se fomentan determinadas capacidades y habilidades -"estas prácticas ayudan a conocer mejor la asignatura al profundizarse en determinados apartados del temario...ayudan a desarrollar la empatía... [y, al mismo tiempo], se conoce mejor a los compañeros de clase", "me ha 
encantado ver como todos los compañeros hemos trabajado juntos, exponiendo ideas y escuchando a los demás y finalmente cómo hemos valorado el trabajo individual de cada uno" o "la simulación sirve porque te obliga a estudiar los temas y repasar asignaturas anteriores, además incentiva la improvisación y la oratoria, a lo que no se le presta mucha atención durante la carrera. Por otro lado, destacaría la elección del aula, ya que en el éxito de la simulación ha influido la posición que teníamos los alumnos...unos frente a otros"- y que, además y para terminar, es una forma muy atractiva de aprendizaje -la "simulación...es una actividad muy productiva, además de divertida. En este tipo de clases, se aprende muchísimo" o "ha sido la clase en la que, además de aprender bastante, mejor nos lo hemos pasado"-.

\section{Ejemplificación de las simulaciones en distintas asignaturas jurídico-laborales}

\subsection{Simulación genérica: proceso de selección de personal}

Como ya se ha anunciado, esta simulación debería ser común a todas las titulaciones; y ello debido a la necesidad, en un mercado de trabajo cada vez más competitivo, de los alumnos -futuros trabajadores- de someterse a arduos y complejos procesos de selección. Ello debiera conllevar que el alumno universitario, aunque sea de esta forma, se acerque, lo más posible, a dichos procesos.

Lo normal y frecuente es que los alumnos presenten enormes carencias en este sentido máxime, cuando muchos de ellos, nunca se han presentado a un proceso de estas características-; de ahí que, previamente, a la simulación se les ofrezca una breve charla sobre aspectos básicos de un proceso de selección como elaboración de currículo, dinámicas, entrevistas, comportamientos, vestuario e, incluso -pero siempre con la suficiente cautela, puesto que no existe unas reglas comunes en todos los procesos de selección-, se les explica el motivo de las pruebas y los objetivos de los seleccionadores.

Tras la clase introductoria, los alumnos confeccionarán sus currículos, que serán entregados con antelación a la realización de la correspondiente simulación. Se indica que para preservar la intimidad y la protección de datos de los alumnos, los currículos pueden ser falsos; eso sí, siempre que respondan, más o menos, a la realidad de un alumno universitario de la correspondiente titulación.

El diseño del proceso se atendrá a las características del puesto de trabajo ofertado, que se corresponderá, lo máximo posible, a la realidad y, por lógica, a la titulación que esté cursando el alumno. De hecho, lo normal es que se tome un anuncio real publicado a través de los mecanismos habituales como prensa escrita y, en los últimos tiempos, fundamentalmente, los buscadores de empleo de internet. Partiendo de ahí, el proceso suele constar de las siguientes fases. Hay que aclarar, antes de continuar, que se trata de una actividad individual; aunque las dinámicas de grupo obliguen a una interacción entre los alumnos mediante la conformación de los correspondientes grupos.

La sesión de la simulación comienza con la criba curricular. Se nombra a los alumnos elegidos para la realización de la actividad, que empieza con la presentación por los "candidatos" de ellos mismos -tienen que argumentar que son los idóneos para el puesto de trabajo- así como de sus currículos ante sus compañeros. Para ello, cuentan con un breve y tasado espacio de tiempo. 
Seguidamente, se realizan, al menos, dos dinámicas de grupo, de las que habitualmente se llevan a cabo en los procesos de selección empresariales. Así, se acude a dinámicas grupales como las siguientes: la construcción con materiales inconexos de la torre más alta y más bonita, la adopción de papeles muy distintos para decidir quién o quiénes tienen que acceder a bunker en caso de guerra, etcétera. Con ellas, se aprecian, entre otras, capacidades como las de posicionarse en distintos roles, de organización, de trabajo en equipo y de liderazgo.

Los alumnos que hayan superado esta última fase -se eligen a tres- pasarán a la entrevista final, que es realizada, como el resto de la actividad, por un profesional de la selección de personal -miembro de un departamento de recursos humanos-. Lo que acerca, aún más, la simulación a la realidad. Tras las entrevistas individuales, y sin presencia en cada una de ellas de los restantes candidatos, se procede a la elección del alumno, que "obtiene" el puesto de trabajo. La simulación finaliza con las preguntas que los alumnos-público realizan al responsable de la actividad. Esto suscita un rico debate, con el que se enriquece enormemente la práctica.

No son pocos los alumnos que, con el paso del tiempo, me han comunicado que esta actividad les sirvió bastante tanto para superar -eso sí, en parte, ya que lo que se jugaban era muy distinto- el miedo a dichos procesos y, principalmente, el temor a intervenir en público, como para conocer cómo funciona y cuáles suelen ser las pruebas, a las que se someten a los candidatos a los puestos de trabajo, por lo que se han enfrentado con mayor conocimiento y comodidad a un procedimiento real.

\subsection{Simulaciones específicas}

En este epígrafe, se van a indicar las especificidades, fundamentalmente de contenido, de las simulaciones realizadas en cada una de las asignaturas impartidas y que, desde un punto de vista formal, responden a la dinámica descrita en un epígrafe precedente.

\section{a) Grado de Derecho}

La asignatura impartida es una optativa denominada Relaciones Laborales en la Empresa. Su contenido condiciona, como no puede ser de otro modo, el objeto de la simulación que, en esta asignatura, ha consistido en dos tipos de negociaciones. En primer lugar, la negociación de diferentes acuerdos de empresa -debe tomarse en consideración que debido al número de alumnos hay que generar diferentes supuestos de hecho-; en unos casos, obedecían a la inaplicación de un convenio colectivo y, en otros, a una modificación del mismo. Cada uno requiere, conforme a los parámetros legales, de diferentes procedimientos, que debían ser seguidos por los alumnos. Asumiendo el rol -empresa y representantes de los trabajadores- conforme al reparto previamente realizado por el profesor. Y en segundo lugar, la negociación de un plan de igualdad dentro de la empresa. En esta ocasión, se ofrece al alumnado los parámetros genéricos de tantas empresas como simulaciones se vayan a realizar. A partir de esa caracterización, se inicia la negociación.

Este año, el total de alumnos ascendía a 30; y todos, menos dos, valoraron positivamente este tipo de actividad, ya que han considerado que se trata de una forma de aprendizaje, que requiere de un mayor compromiso, implicación, responsabilidad y seriedad por parte del alumno pero, al tiempo, es una forma "más divertida" -se cita 
textualmente- de adquirir los correspondientes conocimientos y competencias. Se quiere destacar que cinco alumnos han manifestado que este tipo de actividad les ha permitido vencer el miedo a hablar en público -lo que no habían hecho en los tres años anteriores del grado-. Sólo dos alumnos han mostrado su disconformidad con el modelo; y lo han justificado en el hecho de su dificultad de colaboración con los restantes compañeros y en su apego al sistema de aprendizaje memorístico.

\section{b) Grado de Relaciones Laborales y Recursos Humanos}

En este Grado, la asignatura recibe el nombre de Solución Extrajudicial de Conflictos d Trabajo. En esencia, el contenido de la misma se articula en tres grandes bloques: conflicto laboral, manifestación del conflicto y medidas de solución extrajudicial de conflictos -dentro de este último, se dedica una gran atención a los acuerdos marco de solución extrajudicial de conflictos-. Se trata, por tanto, de una asignatura especialmente favorable a la realización de simulaciones. A ello se une que el número de alumnos, normalmente, no es especialmente alto. Por ejemplo, en el último año, el número ascendió a 12, por lo que la simulación se ha llevado a cabo por toda la clase en un único supuesto; y en este caso, todos los alumnos han mostrado un enorme interés e implicación, lo que ha supuesto que la valoración por el profesor así como por los propios alumnos haya sido enormemente positiva.

La dinámica de estas simulaciones es la descrita en el apartado correspondiente de este trabajo. La única particularidad consiste en que la solución extrajudicial del conflicto de trabajo se somete a las vías institucionalizadas por los interlocutores sociales, ya sea en el ámbito estatal -ASAC-, ya sea en el autonómico -por desarrollarse la actividad en la Comunidad Autónoma Andaluza, se acude al Sistema Extrajudicial de Resolución de Conflictos Laborales de Andalucía (SERCLA)-. Además, en esta ocasión, la simulación va acompañada de la redacción de los documentos exigidos por cada uno de estos Acuerdos así como de la presentación final del acta conforme a las directrices marcadas por los mismos. Por último, esta simulación ha favorecido que se fomente la capacidad para alcanzar acuerdos mediante, fundamentalmente, conciliaciones o mediaciones. Ello ha permitido realizar una actividad muy completa.

\section{c) Grado de Turismo}

La amplitud del temario de la asignatura impartida en este Grado -Legislación Laboral en el Sector Turístico- facilita la posibilidad de decidir el contenido de la simulación. En concreto, se suelen realizar dos consistentes, la primera, en la negociación del convenio provincial de hostelería, y, la segunda, en un juicio por despido.

La primera simulación se lleva a cabo en las primeras semanas de clase tras el conocimiento de qué es y cómo se desarrolla la negociación de convenios colectivos. Las deficiencias jurídicas de estos alumnos se suplen con sus conocimientos económicos y sociales del sector de la hostelería, que les sirven de base para la susodicha negociación.

Por su parte, la simulación del juicio de despido se hace relativizando, por ser alumnos de un Grado no jurídico, los aspectos puramente procesales. Esta acomodación facilita, de manera importante, su realización pero no neutraliza, en absoluto, conocer y 
fomentar las capacidades y habilidades de estos alumnos derivadas de este tipo de actividades.

d) Postgrados: Master de Mediación, Master de Igualdad y Master de Abogacía.

Pese a tenerse docencia en los indicados masteres, tanto en el de abogacía como en el de igualdad, pese a ser muy proclives a llevarse a cabo simulaciones, la escasez de sesiones -dos- impide que éstas se pueda poner en práctica. Aún así, se intenta que haya una actividad, muy breve eso sí, similar a una simulación. Para ello, se acoge el esquema de otras simulaciones como la de juicio o la de negociación de un plan de igualdad respectivamente. Pese a este intento, no se pueden extraer conclusiones. Sin embargo, la simulación en el master de mediación está instituida desde el principio; y a ella se dedica la última sesión del módulo. Dos son sus principales problemas.

En primer lugar, la interdisciplinariedad de este último master. Consecuentemente, los alumnos proceden de distintas titulaciones y, en muy pocas ocasiones, de naturaleza jurídico-laboral. De ahí que el primer hándicap a vencer sea dotar al alumno de un conocimiento básico sobre la realidad jurídica del mundo de las relaciones de trabajo incluido los mecanismos de solución extrajudicial de conflictos laborales-. Se hace imprescindible, por tanto, ese conocimiento para acometer la correspondiente mediación laboral, que es la esencia del módulo. Por ello, se ha preferido, en los últimos años, modificar el contenido de la simulación vaciándolo, en la medida de lo posible, de los aspectos estrictamente jurídicos y, por ende, acercándolo a la realidad más candente y actual de la conflictividad laboral, a fin de que apliquen sus conocimientos previos de contenido psicológico -los primeros módulos de este master son los aspectos sicológicos de la mediación- en la mediación laboral -aunque no puede ocultarse, que ésta presenta ciertas particularidades y diferencias con respecto al planteamiento genérico de la medicación, lo que, en no pocas ocasiones, se convierte, desgraciadamente, en otro problema de este master-. En esta simulación, normalmente, se cuenta, debido al perfil de los alumnos y a los objetivos del master, con la presencia de otros roles; esto es, además de trabajadores, empresarios y órgano extrajudicial, se hace partícipe a organizaciones de consumidores y comerciantes, administraciones o asociaciones vecinales. Por lo demás, se reproduce la dinámica ya conocida.

En segundo lugar, la escasez de sesiones, lo que dificulta bastante la propia simulación; o sea, la falta de tiempo para la adquisición de los conocimientos teóricos así como para la preparación y realización de la simulación. Exactamente, se imparten 15 horas en tres sesiones de 5 horas cada una, las cuales se pueden suceder en la misma semana, es decir, lunes, miércoles y viernes; de tal modo, que, algunos años, se presenta la simulación el primer día -lunes-, cuando todavía el alumnado no tiene ningún conocimiento de la materia, para que se realice el viernes de esa misma semana. Este problema es sistemáticamente puesto de manifiesto por los alumnos en el cuestionario final; y a la misma vez, es de difícil solución, ya que el número y fechas de las clases viene estipulado por las directrices del master.

\section{A modo de conclusiones}

La experiencia docente en materia de simulaciones a lo largo de mis años ha sido particularmente atractiva y enriquecedora, puesto que los objetivos propuestos, en casi su totalidad, han sido alcanzados y, fundamentalmente, se ha logrado que el alumno se 
inserte a la par que interiorice otra dinámica de aprendizaje, en la que, como se ha visto a lo largo de estas páginas, tiene como finalidad no sólo, que también, el conocimiento teórico y dogmático de las correspondientes asignaturas sino, igualmente, adquirir una serie de competencias de gran significación para su desarrollo profesional -y, por qué no, vital-.

Ahora bien, ello requiere ineluctablemente de un cambio de actitudes por los sujetos implicados -alumnos y profesor-, al que no siempre se está dispuesto por razones de tiempo, de carga de trabajo y de disposición. De ahí que resulte clave para la materialización de este aprendizaje, en primer lugar, la voluntad del docente, generando en clase un clima distendido pero formal y respetuoso y, al mismo tiempo, crítico; y, en segundo lugar, un interés del discente por implicarse en una forma, demasiadas veces desconocida para él, de análisis y de aprendizaje, que sobrepasa el mero estudio memorístico y, por ello, mucho más complejo en un triple sentido. Por un lado, mediante un esfuerzo de trabajo mayor; por otro lado, obligándole a realizar un análisis y reflexión, que no siempre forma parte del aprendizaje habitual y, por último, fomentando, actuando e interviniendo en unas esferas -léase, competenciastradicionalmente olvidadas y desatendidas.

Pese a todo y con todo ello, se está ante una enriquecedora y estimulante diversificación del aprendizaje hasta el punto de que se debía institucionalizar como método de trabajo -obviamente con sus correspondientes adaptaciones- en todas las disciplinas de las ciencias jurídicas.

\section{Bibliografía}

GARCÍA BARRERA, A. (2015): "Importancia de la competencia argumentativa en el ámbito educativo: una propuesta para su enseñanza a través del role playing online". En revista Revista de Educación a Distancia, N. 45, marzo de 2015, p. 1-20.

MAYORGA FERNÁNDEZ， Ma . J. y MADRID VIVAR, D. (2010): "Modelos didácticos y estrategias de enseñanza en el Espacio Europeo de Educación Superior". En revista Tendencias Pedagógicas, Vol. 1, N. 15, 2010, pp. 91-111.

ORTÍZ DE URBINA CRIADO, M., MEDINA SALGADO, S., y DE LA CALLE DURÁN, C. (2010): "Herramientas para el aprendizaje colaborativo: una aplicación práctica del juego de rol". En revista Teoría de la Educación. Educación y Cultura en la Sociedad de la Información, Vol. 11, N. 3, 2010, pp. 277-301.

TALÉNS VISCONTI, E. E., (2016): "El aprendizaje cooperativo como vehículo de innovación docente en ciencias jurídicas". En revista Actualidad Jurídica Iberoamericana, N. 4.bis, abril de 2016, pp. 203-216. 